\title{
Basic calcium phosphate deposition disease - a clinical and imaging analysis
}

\author{
Paulina Vele ${ }^{1}$, Laura Otilia Damian², Siao-Pin Simon ${ }^{1,2}$, Ioana Felea ${ }^{2}$, Laura Muntean ${ }^{1,2}$, \\ Ileana Filipescu ${ }^{1,2}$, Maria Magdalena Tamas ${ }^{1,2}$, Cristina Pamfil', \\ Cristian Nedelcut ${ }^{2}$, Simona Rednic ${ }^{1,2}$ \\ ${ }^{1}$ Rheumatology Department, „luliu Hatieganu“ University of Medicine and Pharmacy, Cluj-Napoca, Romania \\ ${ }^{2}$ Rheumatology, Emergency County Teaching Hospital, Cluj-Napoca, Romania
}

\begin{abstract}
Background. Basic calcium phosphate (BCP) deposition disease is a frequent musculoskeletal problem characterized by the intraarticular or periarticular deposition of carbonate substituted hydroxyapatite, octacalcium phosphate and tricalcium phosphate. BCP and calcium pyrophosphate (CPP) crystals play an important role in the pathogenesis of osteoarthritis.

Objectives. The primary aim was to evaluate the clinical and ultrasonographic characteristics of patients with $\mathrm{BCP}$. The secondary aim was to compare the radiographic scores of patients with $\mathrm{BCP}$, calcium pyrophosphate dihydrate (CPPD) deposition disease and degenerative disease (DD).

Material and methods. 50 patients with BCP deposition disease diagnosed by imaging or by identification of BCP in synovial fluid (SF) were included in the study. The second part of the study included 20 patients with BCP crystals in SF, 20 patients with CPP crystals in SF and 20 patients with degenerative changes on radiography and without crystals in SF. Clinical, ultrasonographic and radiographic data were recorded.

Results. The shoulder joint (56\%), followed by the knee joint (36\%) and the acute clinical presentation (84\%) were the most common findings. The fragmented ultrasound appearance (54\%) was the most frequent, followed by the arch-shaped (36\%). The localization of the deposits was found most often in the tendons (68\%), mainly in the supraspinatus tendon (55.88\%). Higher K/L scores were found in patients with BCP and CPP crystals than in the patients with $\mathrm{DD}$. K/L score $\geq 3$, defining osteoarthritis was associated with the presence of $\mathrm{BCP}$ and CPP crystals.

Conclusions. The shoulder and the fragmented ultrasound pattern are the most common findings in patients with BCP. Higher K/L scores are found in patients with BCP and CPP crystal associated disease than in the degenerative disease patients.
\end{abstract}

Keywords: basic calcium phosphate, calcium pyrophosphate, osteoarthritis, Kellgren-Lawrence

\section{INTRODUCTION}

Basic calcium phosphate (BCP) deposition disease is defined as the deposition of BCP crystals intraarticular or periarticular. BCP crystals are containing carbonate substituted hydroxyapatite, octacalcium phosphate. From a clinical point of view, the deposits can be asymptomatic, or with an acute or chronic presentation. The shoulder is the most affected joint, mainly the rotator cuff, followed by the hips. Intraarticular deposition of crystals leads to a destructive arthropathy, known as Milwaukee shoulder (1-4). The BCP crystals are formed when extracellular inorganic pyrophosphate (PPi) is low, mainly when inorganic phosphate $(\mathrm{Pi}) / \mathrm{PPi}$ rapport is over
$70(5,6)$. The crystals have a complex effect on chondrocytes, macrophages and fibroblasts and also have the capacity to activate the nucleotide-binding domain, leucine-rich-containing family, pyrin domain-containing-3 (NLRP3) inflammasome. These mechanisms lead to inflammation, articular destruction, which can finally lead to osteoarthritis (3,711). The gold standard for diagnosis is the identification of crystals in synovial fluid (SF), but due to the small dimensions of the crystals and tendance to form aggregates are difficult to be identified by light microscopy. Novel techniques are available but are expensive and complex (12). The calcifications are frequently discovered by radiological examination, 
having different sizes and different appearances $(13,14)$. Ultrasound (US) is also a valuable method for the identification of BCP deposits (15-17).

This study aimed to describe clinical and imaging characteristics of patients with BCP. Our secondary aim was to compare the Kellgren-Lawrence score $(\mathrm{K} / \mathrm{L})$ score in patients with $\mathrm{BCP}$, calcium pyrophosphate dihydrate (CPPD) deposition disease and degenerative disease (DD).

\section{MATERIAL AND METHODS}

In this transversal study were included patients with BCP crystals in SF or with evidence of BCP (radiographic or by US). Patients with gout or other inflammatory rheumatic diseases were excluded from the study. Data about age (years), sex, number of tenders (TJC) and swollen joints (SJC), erythrocyte sedimentation rate (ESR) and C-reactive protein (CRP), the affected joints, clinical presentation as acute or chronic were recorded. US examination was performed using an Esaote My Lab 25 Gold with 5-10 and 10-18 MHz linear probes according to musculoskeletal guidelines. The protocol included searching for calcific deposits in symptomatic joints, identification of US pattern of calcification described for BCP (arch-shaped, fragmented, nodular, cystic), the affected joint, and the localization of deposits in the tendon, intraarticular, bursae, recess or other tissues (15-19).

BCP subgroup: A subgroup of 20 patients with BCP in SF was compared with 20 patients with CPPD and with 20 patients with DD. All the diseases were at the knee level.

CPPD group: Patients who fulfilled McCarty criteria for CPPD (imaging evidence of CPPD and crystals in SF) were included in the study (20).

$D D$ group: Patients with degenerative changes on radiography and without crystals on SF analysis were consecutively included in the study.

Age, TJC, SJC, the visual analogue scale for pain (VAS), ESR (mm/h), CRP (mg/dl) were recorded for these three groups. All patients from the BCP subgroup, CPPD and DD group underwent radiographs in posteroanterior, weight-bearing semi-flexed view. All radiographs were graded according to $\mathrm{K} / \mathrm{L}$ score (21). The score was interpreted at both knees, but also a medium $\mathrm{K} / \mathrm{L}$ score, as a medium between the left and the right knee score was calculated. Osteoarthritis was defined as a K/L score $\geq 3$. BCP and calcium pyrophosphate $(\mathrm{CPP})$ crystals were identified in the SF according to the description of crystals $(12,22)$.

All patients included in the study consented for participation and the approval of the University Ethics Committee was obtained.

Statistical analysis was performed using Microsoft Excel. We considered a level of $\mathrm{p}<0.05$ statistically significant.

\section{RESULTS}

The study included 50 patients with BCP deposition disease. The characteristics of the study population are presented in Table 1 .

TABLE 1. General characteristics of the study population with $B C P$

\begin{tabular}{|l|c|}
\hline Variable & $\mathbf{n = 5 0}$ \\
\hline Age (years) & $62.28 \pm 10.8$ \\
\hline Sex ratio of male:female & $17: 33$ \\
\hline TJC & $1.92 \pm 1.9$ \\
\hline SJC & $1.08 \pm 0.6$ \\
\hline ESR $(\mathrm{mm} / \mathrm{h})$ & $20.1 \pm 19.8$ \\
\hline CRP $(\mathrm{mg} / \mathrm{dl})$ & $1.8 \pm 4.5$ \\
\hline Shoulder & $28(56 \%))$ \\
\hline Knee & $18(36 \%)$ \\
\hline Hand & $5(10 \%)$ \\
\hline Hip & $2(4 \%)$ \\
\hline Elbow & $2(4 \%)$ \\
\hline Ankle & $1(2 \%)$ \\
\hline Acute presentation & $42(84 \%)$ \\
\hline Chronic presentation & $8(16 \%)$ \\
\hline
\end{tabular}

TJC-tender joint count; SJC- swollen joint count; ESR-erythrocyte sedimentation rate; $C R P-C$ reactive protein; $B C P$-basic calcium phosphate

The second, fragmented pattern was the most frequent in patients with BCP (Table 2). Regarding the location, the tendon was the most affected location and the supraspinatus the most common implicated (Table 2). The fragmented appearence was more common in patients with acute presentation and monoarticular involvement (Figure 1).

TABLE 2. US patterns and location of the BCP deposits

\begin{tabular}{|l|c|}
\hline Variable & N (\%) \\
\hline Calcification pattern & $\mathbf{N = 5 0}$ \\
\hline I Arch-shaped & $18(36 \%)$ \\
\hline II Fragmented & $27(54 \%)$ \\
\hline III Nodular & $5(10 \%)$ \\
\hline IV Cystic & $0(0 \%)$ \\
\hline BCP deposits localization & $\mathbf{N = 5 0}$ \\
\hline Tendon & $34(68 \%)$ \\
\hline Bursae & $2(4 \%)$ \\
\hline Intraarticular & $6(12 \%)$ \\
\hline
\end{tabular}




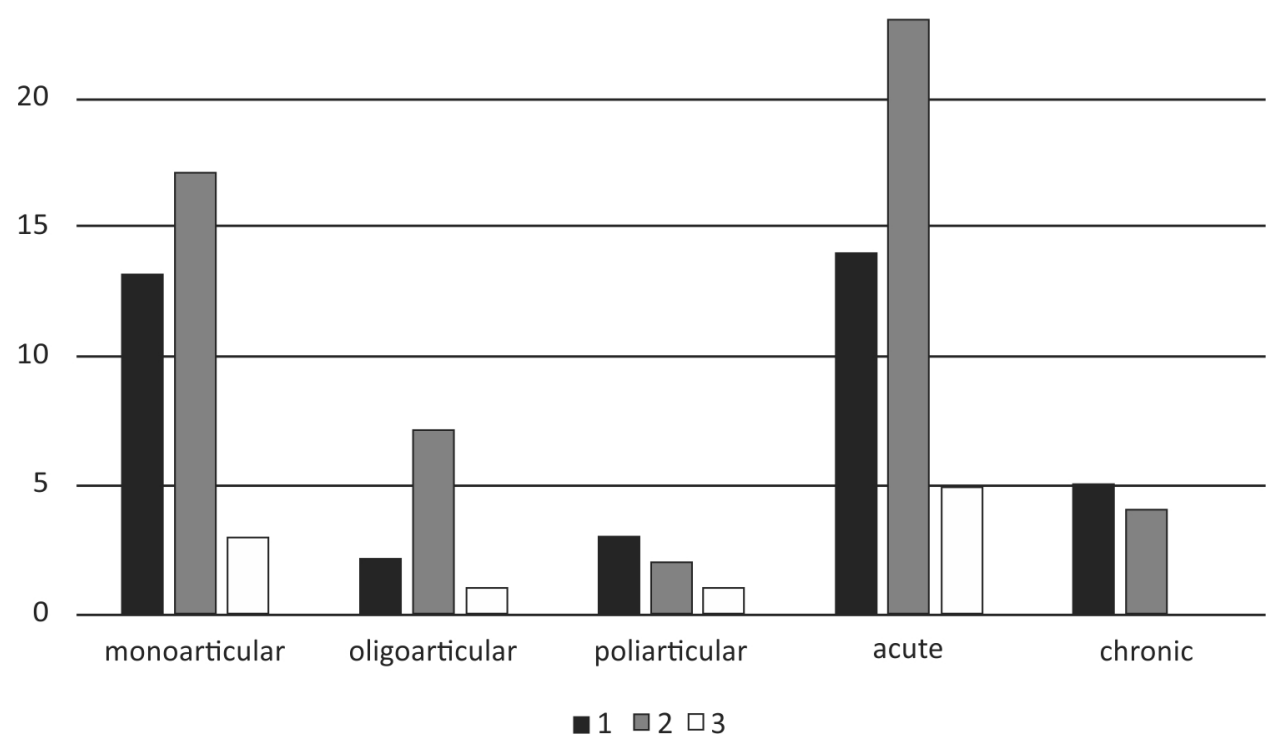

FIGURE 1. US patterns according to clinical characteristics

\begin{tabular}{|l|c|}
\hline Variable & N (\%) \\
\hline Recess & $6(12 \%)$ \\
\hline Bursae and recess & $2(4 \%)$ \\
\hline Tendons & $\mathbf{N = 3 4}$ \\
\hline Supraspinatus tendon & $19(55.88 \%)$ \\
\hline Quadriceps tendon & $10(29.41 \%)$ \\
\hline Hand extensors tendons & $5(14.7 \%)$ \\
\hline
\end{tabular}

Values are expressed as number of patients or joints (percentage)

\section{BCP subgroup, CPPD and DD group evaluation}

Comparing the three groups, significant differences were found between BCP, CPPD and DD regarding TJC, SJC, VAS and ESR. The results are described in detail in Table 3 .

TABLE 3. General characteristics of the patients with $B C P, C P P D$ and $D D$

\begin{tabular}{|l|c|c|c|c|}
\hline Variable & $\begin{array}{c}\mathbf{B C P} \\
\mathbf{n = 2 0}\end{array}$ & $\begin{array}{c}\text { CPPD } \\
\mathbf{n = 2 0}\end{array}$ & $\begin{array}{c}\text { DD } \\
\mathbf{n = 2 0}\end{array}$ & $\mathbf{p}$ \\
\hline Age (years) & $61.6 \pm 138$ & $61.1 \pm 109$ & $60 \pm 53$ & 0.8 \\
\hline TJC & $2.85 \pm 2.7$ & $4.35 \pm 3.6$ & $1.8 \pm 0.5$ & 0.01 \\
\hline SJC & $1.4 \pm 0.5$ & $1.8 \pm 0.9$ & $0.35 \pm 0.5$ & 0.0005 \\
\hline VAS & $7.7 \pm 1.6$ & $7.8 \pm 1.05$ & $5.7 \pm 1.9$ & 0.001 \\
\hline $\operatorname{ESR~}(\mathrm{mm} / \mathrm{h})$ & $26.4 \pm 59.5$ & $22.8 \pm 37.2$ & $11.1 \pm 41$ & 0.002 \\
\hline CRP $(\mathrm{mg} / \mathrm{dl})$ & $1.5 \pm 8$ & $0.6 \pm 0.6$ & $0.6 \pm 0.7$ & 0.1 \\
\hline
\end{tabular}

TJC-tender joint count; SJC- swollen joint count; VAS-visual analogue scale; ESR-erythrocyte sedimentation rate; CRP-C reactive protein; $B C P$-basic calcium phosphate; CPPD-calcium pyrophosphate dihydrate; $D D$-degenerative disease

Significant higher K/L scores were found in patients with crystal disease than in patients with DD at the right and left knee, but also when was calculated medium K/L score (Table 4). Between the BCP and
CPPD group wasn't found any statistical significance $(\mathrm{p}=0.89$ for medium $\mathrm{K} / \mathrm{L}$ score, $\mathrm{p}=0.9$ for right knee and $\mathrm{p}=0.6$ for the left knee.

TABLE 4. $K / L$ score of the patients with $B C P, C P P D$ and $D D$

\begin{tabular}{|l|c|c|c|c|}
\hline Knee K/L score & BCP & CPPD & DD & p value \\
\hline Right & $2.55 \pm 1.5$ & $2.6 \pm 1.4$ & $1.2 \pm 0.9$ & $\mathrm{P}=0.0002$ \\
\hline Left & $2.35 \pm 1.8$ & $2.55 \pm 1.2$ & $1.4 \pm 1.3$ & $\mathrm{P}=0.0008$ \\
\hline Medium score & $2.45 \pm 1.4$ & $2.57 \pm 1.1$ & $1.3 \pm 0.9$ & $\mathrm{P}=0.0007$ \\
\hline
\end{tabular}

K/L - Kellgren-Lawrence

$\mathrm{K} / \mathrm{L}$ score $\geq 3$ was associated with the presence of BCP (4.88 [1.1-19.9] and CPPD (OR 12 [95\% CI 2.7-53], presented in Table 5.

TABLE 5. Osteoarthritis in patients with $B C P, C P P D$ and $D D$

\begin{tabular}{|l|l|l|l|}
\hline Variable & $\begin{array}{l}\text { Medium K/L } \\
\text { score }<\mathbf{3}\end{array}$ & Medium K/L $\geq 3$ & OR (95\% CI) \\
\hline BCP & 9 & 11 & $4.88(1.1-19.9)$ \\
\hline DD & 16 & 4 & \multirow{2}{*}{$12(2.7-53)$} \\
\hline CPPD & 5 & 15 & \\
\hline DD & 16 & 4 & $0.4(0.1-1.5)$ \\
\hline BCP & 9 & 11 & \\
\hline CPPD & 5 & 15 & \\
\hline
\end{tabular}

$K / L$ - Kellgren-Lawrence, BCP-basic calcium phosphate; CPPD-calcium pyrophosphate dihydrate; $D D$-degenerative disease

\section{DISCUSSION}

The first part of the study examined the clinical and US characteristics of patients with BCP. Similar to other studies the shoulder was the most frequent 
joint involved and the clinical presentation was acute and monoarticular $(3,4)$. The second most affected joint in our study was the knee, in contradiction with other studies in which predominant was the hip joint $(2,4)$. The second, fragmented US pattern was the most frequent, probably because all patients were symptomatic and is known that the resorptive phase is associated with pain (15-17). In concordance with other studies, the most frequent localization of the deposits is in the supraspinatus tendon $(3,4)$.

The second part of the study analyzed K/L score of three groups with BCP, CPPD and DD. BCP and CPP crystals are found in the SF of patients with osteoarthritis $(23,24)$. Fuerst et al. found BCP crystals in all patients undergoing joint replacement for severe osteoarthritis (24). In clinical practice, many patients remain underdiagnosed due to difficulty to identify crystal with usual techniques (12). Both of these calcium-containing crystals induces inflammation and joint damage through complex mechanisms on chondrocytes and other cells. Also, CPP and BCP crystals have the property of activating the inflammasome NLRP3 $(3,8-10,25,26)$. For BCP are described other pathways too: increase the production of matrix metalloproteinases, tumour necrosis factor-alpha, interleukin 6 and Prostaglandin E2 (3,7$10,26)$. In the phatogenesis of osteoarthitris are implicated many factors. One of the most controversial is inflammation, mainly because osteoarthritis is not a disease characterized by inflammation (11,27-29).

\section{REFERENCES}

1. Garcia GM, McCord GC, Kumar R. Hydroxyapatite crystal deposition disease. Semin Musculoskelet Radiol. 2003;7(3):187-93

2. Rosenthal AK. Basic calcium phosphate crystal-associated musculoskeletal syndromes: An update. Curr Opin Rheumatol. 2018;30(2):168-172

3. McCarthy GM, Dunne A. Calcium crystal deposition diseases beyond gout. Nat Rev Rheumatol. 2018;14(10):592-602.

4. Beckmann NM. Calcium Apatite Deposition Disease: Diagnosis and Treatment. Radiol Res Pract. 2016;4801474.

5. Thouverey C, Bechkoff G, Pikula S et al. Inorganic pyrophosphate as a regulator of hydroxyapatite or calcium pyrophosphate dihydrate mineral deposition by matrix vesicles. Osteoarthr Cartil. 2009;17(1):64-72.

6. Johnson K, Terkeltaub R. Inorganic pyrophosphate (PPI) in pathologic calcification of articular cartilage. Front Biosci. 2005;10:988-97.

7. Morgan MP, Whelan LC, Sallis JD et al. Basic Calcium Phosphate Crystal-Induced Prostaglandin E2 Production in Human Fibroblasts: Role of Cyclooxygenase 1, Cyclooxygenase 2, and Interleukin-1 $\beta$. Arthritis Rheum. 2004;50(5):1642-9.

8. Nadra I, Mason JC, Philippidis P et al. Proinflammatory activation of macrophages by basic calcium phosphate crystals via
From a clinical point of view, the Milwaukee shoulder can sustain that inflammatory pathway.

Our results found higher values of TJC, SJC and VAS in the crystal associated groups than in the DD group. Similar results were found in a previous study in which was compared CPPD with osteoarthritis patients and in another study regarding pain, but not with arthritis (30,31). In our study significant higher $\mathrm{K} / \mathrm{L}$ scores were found in patients with $\mathrm{BCP}$ and CPPD than in patients with DD, but not between the crystal associated groups. Also, K/L score $\geq 3$, which defines osteoarthritis was associated with the presence of BCP and CPPD. Higher radiographic scores were correlated to the presence of BCP crystals in a study by Derfus et al. too (32). In a recent study comparing the presence of $\mathrm{CPP}$ and $\mathrm{BCP}$ crystals in patients with osteoarthritis according to $\mathrm{K} / \mathrm{L}$ score, there was not found any statistical significance between groups (33). Also, some studies found an association between osteoarthritis and chondrocalcinosis $(34,35)$. The small number of patients and the absence of a structural phenotype analysis of osteoarthritis is the main limitation of the study.

\section{CONCLUSION}

The shoulder and the knee joint are the most affected joints. The fragmented US pattern is the most common and associated with an acute clinical presentation. Higher radiographic scores are associated with BCP and CPP crystal disease.

Conflict of interest: none declared Financial support: none declared

protein kinase $C$ and MAP kinase pathways: $A$ vicious cycle of inflammation and arterial calcification? Circ Res. 2005; 96(12):1248-56.

9. Cunningham CC, Mills E, Mielke LA et al. Osteoarthritis-associated basic calcium phosphate crystals induce pro-inflammatory cytokines and damage-associated molecules via activation of Syk and PI3 kinase. Clin Immunol. 2012;144(3):228-36.

10. Jin C, Frayssinet $P$, Pelker R et al. NLRP3 inflammasome plays a critical role in the pathogenesis of hydroxyapatite-associated arthropathy. Proc Natl Acad Sci USA. 2011;108(36):14867-72.

11. Conway R, McCarthy GM. Calcium-Containing Crystals and Osteoarthritis: an Unhealthy Alliance. Curr Rheumatol Rep. 2018; 20(3):13.

12. Yavorskyy A, Hernandez-Santana A, McCarthy G et al. Detection of calcium phosphate crystals in the joint fluid of patients with osteoarthritis - Analytical approaches and challenges. Analyst. 2008;133(3):302-18.

13. Omoumi $P$, Zufferey $P$, Malghem J et al. Imaging in Gout and Other Crystal-Related Arthropathies. Rheum Dis Clin of North Am. 2016;42(4):621-644.

14. Jacques T, Michelin P, Badr S et al. Conventional Radiology in Crystal Arthritis: Gout, Calcium Pyrophosphate Deposition, and 
Basic Calcium Phosphate Crystals. Radiol Clin of North Am. 2017;55(5):967-984.

15. Chiou HJ, Chou YH, Wu JJ et al. Evaluation of calcific tendonitis of the rotator cuff: Role of color Doppler ultrasonography. $J$ Ultrasound Med. 2002;21(3):289-95.

16. Halverson PB. Crystal deposition disease of the shoulder (including calcific tendonitis and milwaukee shoulder syndrome). Curr Rheumatol Rep. 2003;5:244-7.

17. Le Goff B, Berthelot JM, Guillot $P$ et al. Assessment of calcific tendonitis of rotator cuff by ultrasonography: Comparison between symptomatic and asymptomatic shoulders. Joint Bone Spine. 2010;77(3):258-63.

18. Möller I, Janta I, Backhaus M et al. The 2017 EULAR standardised procedures for ultrasound imaging in rheumatology. Ann Rheum Dis. 2017;76(12):1974-1979.

19. Backhaus M, Burmester GR, Gerber T et al. Guidelines for musculoskeletal ultrasound in rheumatology. Ann Rheum Dis. 2001;60:641-9.

20. McCarty DJ. Calcium pyrophosphate dihydrate crystal deposition disease. Arthritis Rheum. 1976;19(3 S):275-85.

21. Kellgren J, Lawrence J. Radiological assessment of osteoarthritis. Ann Rheum Dis. 1957;16(4):494.

22. Swan A, Amer H, Dieppe P. The value of synovial fluid assays in the diagnosis of joint disease: a literature survey. Ann Rheum Dis. 2002;61(6):493-8.

23. Oliviero F, Scanu A, Galozzi P et al. Prevalence of calcium pyrophosphate and monosodium urate crystals in synovial fluid of patients with previously diagnosed joint diseases. Joint Bone Spine. 2013;80(3):287-90.

24. Fuerst M, Bertrand J, Lammers L et al. Calcification of articular cartilage in human osteoarthritis. Arthritis Rheum. 2009; 60(9):2694-703.

25. 25. James SL, Abate D, Abate KH et al. Global, regional, and national incidence, prevalence, and years lived with disability for
354 Diseases and Injuries for 195 countries and territories, 19902017: A systematic analysis for the Global Burden of Disease Study 2017. Lancet. 2018;392(10159):1789-1858.

26. Ea HK, Chobaz V, Nguyen Cet al. Pathogenic Role of Basic Calcium Phosphate Crystals in Destructive Arthropathies. PLoS One. 2013;8(2):e57352.

27. Felson DT. Clinical practice. Osteoarthritis of the knee. $N$ Engl $J$ Med. 2006;23;354(8):841-8.

28. Sokolove J, Lepus CM. Role of inflammation in the pathogenesis of osteoarthritis: Latest findings and interpretations. Ther $A d v$ Musculoskelet Dis. 2013;5(2):77-94.

29. Rosenthal AK. Crystals, inflammation, and osteoarthritis. Curr Opin Rheumatol. 2011;23(2):170-3.

30. Vele P, Damian L, Simon SP et al. Clinical and radiographic findings in patients with chondrocalcinosis. Rom J Rheumatol. 2018;27(2):73-7.

31. Han BK, Kim W, Niu J et al. Association of Chondrocalcinosis in Knee Joints With Pain and Synovitis: Data From the Osteoarthritis Initiative. Arthritis Care Res. 2017;69(11):1651-8.

32. Derfus BA, Kurian JB, Butler JJ et al. The high prevalence of pathologic calcium crystals in pre-operative knees. J Rheumatol. 2002:29(3):570-4.

33. Frallonardo P, Ramonda R, Peruzzo L et al. Basic calcium phosphate and pyrophosphate crystals in early and late osteoarthritis: Relationship with clinical indices and inflammation. Clin Rheumatol. 2018;37(10):2847-2853.

34. Abhishek A, Doherty S, Maciewicz RA et al. Does Chondrocalcinosis Associate with a Distinct Radiographic Phenotype of Osteoarthritis in Knees and Hips? A Case-Control Study. Arthritis Care Res. 2016;68(2):211-6.

35. Abhishek A, Doherty S, Maciewicz R et al. Chondrocalcinosis is common in the absence of knee involvement. Arthritis Res Ther. 2012;14(5). 